\title{
The Application of Transparent Glass Sponges for Improvement of Light Distribution in Photobioreactors
}

\author{
Anna Jacobi ${ }^{1 *}$, Ethel Claudia Bucharsky², Karl Guenter Schell², Peter Habisreuther ${ }^{3}$, Rainer Oberacker ${ }^{2}$, Michael J. Hoffmann², Nikolaos \\ Zarzalis $^{3}$ and Clemens Posten ${ }^{1 *}$ \\ ${ }^{1}$ Karlsruhe Institute of Technology KIT, Institute of Engineering in Life Sciences, Department of Bioprocess Engineering, Fritz-Haber-Weg 2, Building 30.44, 76131 \\ Karlsruhe, Germany \\ ${ }^{2}$ Karlsruhe Institute of Technology KIT, Institute for Applied Materials - Ceramics in Mechanical Engineering, Haid-und-Neu-Str. 7, 76131 Karlsruhe, Germany \\ ${ }^{3}$ Karlsruhe Institute of Technology KIT, Engler-Bunte-Institute, Division of Combustion Technology, Engler-Bunte-Ring 1, Building 40.11, 76128 Karlsruhe, Germany
}

\begin{abstract}
A new concept for improving light dilution and light distribution in photobioreactors by applying transparent, openpored sponges was realized during cultivation experiments. A manufacturing process based on the polymer replica technique was established. A polyurethane template is impregnated with a nanoscaled $\mathrm{SiO}_{2}$ powder suspension (solids loading of $60 \mathrm{wt} \%$ at around $\mathrm{pH} 10$ ) and dried. The green body is prepared by burning-out the polymer at $800^{\circ} \mathrm{C}$. Subsequently the sintering of the remaining $\mathrm{SiO}_{2}$ structure to transparent cellular bodies is carried out at $1330^{\circ} \mathrm{C}$. Many different factors influence the process. The slurry stabilization (viscosity, zeta-potential, $\mathrm{pH}$ ) is important to generate a stable and self-supporting $\mathrm{SiO}_{2}$ shell around the template and achieve appropriate green bodies. The sintering temperature determines the transition of amorphous (transparent) into crystalline silica. The 8 ppi sponge has a porosity of 0.9 , the specific surface area of $568 \mathrm{~m}^{-1}$ means an increase of the (inner) surface to volume ratio of the reactor. Porous glass has a very high surface to volume ratio (about $0.26 \mathrm{~m}^{2} / \mathrm{g}$ of glass at $10 \mu \mathrm{m}$ pore size). Because of multiple and complex reflection of light into the porous glass, the algae can obtain light energy at any location of the total volume. First cultivation experiments in special designed Miniplate reactors show an increase in growth rate (about $+25 \%)$ at low cell densities. The photo conversion efficiency was enlarged from $4.9 \%$ for the empty reactor to $5.6 \%$ and $5.9 \%$ for the 8 and 15 ppi sponge filled reactor. The effect and the improved efficiency of biomass build-up per applied light will be more apparent at high cell densities. Therefore concentration of the standard Tris-Phosphate (TP) medium $(2.5$ fold $)$ and feeding are necessary.
\end{abstract}

Keywords: Microalgae; Photo-bioreactor; Bioprocess design; Growth kinetics; Photo conversion efficiency; Light distribution; Light dilution; Transparent open-pored sponge

Abbreviations: CFD: Computational fluid dynamics; LM: Light microscopy; MRI: Magnetic resonance imaging; OD: Optical density; $\mathrm{P}_{\mathrm{A}}$ : Areal productivity; $\mathrm{P}_{\mathrm{V}}$ : Volumetric productivity; PAR: Photosynthetic active radiation; PCE: Photo conversion efficiency; ppi: Pores per inch; RMS: Root mean square; SEM: Scanning electron microscopy; SFR: Surface to footprint ratio; SVR: Surface to volume ratio; TAP: Tris Acetate Phosphate medium; TP: Tris Phosphate medium; XRD: X-ray diffraction; $\mu$ : Growth rate

\section{Introduction}

Decreasing oil resources force the interest in renewables for biofuel production as well as systems for production of raw materials in chemical industry and high-value products [1-4]. For this purpose phototrophic processes are advantageous using light as energy and carbon dioxide as carbon source for biomass formation with only little additional mineral nutrient necessary. Besides higher land plants like sugarcane, maize, canola and corn, algae show high potential because of higher growth rates and productivities as well as higher photosynthetic efficiencies (efficiency of biomass build-up from sunlight energy) [5-7].

Microalgae are aquatic unicellular and simple multicellular, microbial organisms. They perform no cell differentiation and the lack of additional supporting structures made of structural biopolymers like roots, stems and leaves in land-based plants enhances the photosynthetic efficiency [8]. When grown in photobioreactors cultivation is also possible in arid regions (possible land coverage) and not in competition with food production. Faster growth rates and more generation cycles during the year are advantageous. Furthermore the possible recycle of water and unconsumed nutrients and the tolerance to a high
$\mathrm{CO}_{2}$ content in the gas phase which additionally increases growth kinetics make algae cultivations attractive compared to conventional agriculture.

Cultivation of microalgae in photobioreactors is state of the art and compared to open ponds highly controlled conditions can be applied (temperature, $\mathrm{pH}$, aeration rate and $\mathrm{CO}_{2}$ content, mixing, nutrients and additional feeding). However, not much large scale applications are in operation now, the few that exist produce high value products and no bulk materials e.g. for energetic use. Reasons for that are the high costs and the high demand of auxiliary energy e.g. for mixing. Although closed reactors are already better in light distribution compared to open ponds, there is still a need to reach the goal of reaching a homogeneous light distribution in the medium without the aforementioned disadvantages. Many different geometric modifications exist (e.g. flat plate or tubular reactors, airlift systems,...) all trying to overcome the problem of insufficient light supply for photosynthesis, but no optimal configuration has already been developed. The main problem of

*Corresponding author: Clemens Posten, Karlsruhe Institute of Technology KIT, Institute of Engineering in Life Sciences, Department Bioprocess Engineering, Fritz-Haber-Weg 2, Building 30.44, 76131 Karlsruhe, Germany, Tel: +49 721 60845200; Fax: +49 721 60845202; E-mail: clemens.posten@kit.edu

Received December 07, 2011; Accepted January 20, 2012; Published January 23, 2012

Citation: Jacobi A, Bucharsky EC, Schell KG, Habisreuther P, Oberacker R Hoffmann MJ, et al. (2012) The Application of Transparent Glass Sponges for Improvement of Light Distribution in Photobioreactors. J Bioprocess Biotechniq 2:113 doi: 10.4172/2155-9821.1000113

Copyright: ( 2012 Jacobi A, et al. This is an open-access article distributed unde the terms of the Creative Commons Attribution License, which permits unrestricted use, distribution, and reproduction in any medium, provided the original author and source are credited. 
phototrophic processes is the demand of light as energy source which is not miscible as other substrates. Only low biomass concentrations are achievable due to the inadequate light supply of the whole culture. The light attenuation shows a very fast exponential decay above the layer thickness because of light absorption and scattering by the algae. Large volume elements offer only light limiting conditions resulting in no/less photosynthetic growth whereas on the illuminated surface light saturation or also photo inhibition occurs. So light dilution on the illuminated surface is one issue and diminishing the gradient in the bulk phase is another.

To date many different technical solutions have been described in literature all having the aim to improve light supply, e.g. horizontal and vertical tubular reactors in many different configurations or flat plate reactors with a small layer thickness also in special arrangements. Moreover specific configurations like the Flat Panel Airlift reactor (Subitec) or cheap low-ceiling designs made of plastic foils (Soilx and Proviron) [9-11] are proposed. All this configurations try to improve the surface to volume ratio (SVR) of the photobioreactor as well as the surface to footprint ratio (SFR). Both aspects intend to distribute the light over a larger reactor surface and avoid dark volume elements and to use the incident sunlight as efficient as possible (volumetric and areal productivity $\mathrm{P}_{\mathrm{V}}$ and $\mathrm{P}_{\mathrm{A}}$ ). An optimization of the incident angle of sunlight hitting the reactor surface is a further option for possible developments [10-12].

The concept of this new approach is the enlargement of the inner surface area of the reactor which can contribute to illumination, light distribution and light supply. For this purpose transparent sponges made of glass are employed. In the investigation presented here they are placed in special designed Miniplate reactors for testing the effect. Maximum growth rates $\mu$ and the photo conversion efficiency (PCE) which means the efficiency of buildup of biomass from captured light are determined. Transparent glass sponges are a new class of materials. They are prepared by the polymer replica technique $[13,14]$ employing polyurethane sponges with cell sizes of 8 and 15 pores per inch as templates and commercial available nanoscaled $\mathrm{SiO}_{2}$ powders.

Necessary conditions for obtaining transparent open-celled glass sponges were presented in [13] where topics such as slurry stabilization, temperature for burning-out the polymer and subsequent sintering of the remaining $\mathrm{SiO}_{2}$ structure to transparent cellular bodies are discussed. It was found, that concentrated suspensions at around $\mathrm{pH}$ 10 offer suitable properties for both, bringing enough particles onto the support for the formation of a stable and self-supporting $\mathrm{SiO}_{2}$ shell and for successful sintering of the particulate framework to transparent bodies. Therefore, an adjusted burning-out process and an adequate sintering regime is necessary.

In order to obtain information about the developed phases as a function of sintering temperature-in particular the transition of amorphous into crystalline silica-X-Ray diffractometry was performed. Beside the stability and transparency of the manufactured open-celled sponges, for the successful use of the cellular material, the knowledge of its geometrical properties is necessary. In this study, these parameters are derived from calculations based on datasets of magnetic resonance imaging. Volume Image Analysis on the basis of magnetic resonance imaging (MRI) of porous media has been used now for several decades beginning with the aim of characterizing soil material, e. g. sandstones [15]. Increased resolution has enabled even a tomographic insight into such materials together with the possibility to analyze the structure with respect to geometrically characteristic parameters [16]. Its application to reticulated structures can therefore give the most extended view into its details and has even been used recently to generate high resolution geometrical representations for computational fluid dynamics (CFD) calculations [17-19]. Additionally, scanning electron microscopy (SEM) and light microscopy (LM) were used for the characterization of the resulting transparent sponge structure.

\section{Materials and Methods}

\section{Manufacturing and characterization of transparent sponges}

Nanoscaled amorphous $\mathrm{SiO}_{2}$ particles (AEROSIL OX50, SA of 50 $\mathrm{m}^{2} / \mathrm{g}$, Evonik Degussa GmbH, Essen, Germany) were dispersed in an aqueous media with a solids loading of $60 \mathrm{wt} \%$. The $\mathrm{pH}$ of the slurries was adjusted at $\mathrm{pH} 10$ employing $1 \mathrm{M}$ tetramethylammonium hydroxide (TMAH, AlfaAesar, Karlsruhe, Germany). Details and characterization of suitable suspensions for the preparation of transparent sponges are described in [13].

As templates for the replica technique sponges (having 8 or 15 pores per inch (ppi), Foam Partner Fritz Nauer AG, Leverkusen, Germany) with dimensions $64 \times 122 \times 25 \mathrm{~mm}^{3}$, necessary to cover half of the reactor volume, were used. The polymeric sponges were dip-coated employing well-dispersed slurries and compressed while submerged in order to fill all the pores. The impregnated polyester support was then removed from the slurry and the excess material was squeezed from the foams by means of a rolling mill. The coated polymers were dried for $24 \mathrm{~h}$ at $40^{\circ} \mathrm{C}$ and $80 \%$ humidity. For burning-out the polymer, the coated preforms were placed in an alumina crucible and heated in a furnace (VMK 135, Linn High Therm GmbH, Eschenfelden, Germany) under an air flow rate of approximately $150-200 \mathrm{~L} / \mathrm{h}$. To allow the gradual decomposition and diffusion of the polymeric material, heating was performed carefully up to $800^{\circ} \mathrm{C}$. Subsequently, the samples were sintered at air atmosphere in a chamber furnace (Nabertherm $\mathrm{GmbH}$, Lilienthal, Germany) at $1330^{\circ} \mathrm{C}$ with heating rates of $30 \mathrm{~K} / \mathrm{min}$ and a dwell time of $10 \mathrm{~min}$.

Characterization: For the determination of phase composition after thermal treatments, $\mathrm{X}$ ray diffraction (XRD) spectra were recorded using a Siemens D500 diffractometer, operating with CuKa radiation at $40 \mathrm{kV}$ and $25 \mathrm{~mA}$. Macroscopic analysis was performed by image analysis using a digital camera integrated on an optical microscope (Olympus AX70, Pittsburgh, USA). In addition, volume image analysis was performed using magnetic resonance imaging (MRI). In the present work the ${ }^{1} \mathrm{H}$-MRI measurements were taken on a BrukerAvance 200 SWB tomograph (magnetic flux density, 4.7 T; micro-2.5 gradient system generating up to $1 \mathrm{~T} / \mathrm{m}$ ). Therefore, the sintered transparent sponge samples were cut into pieces of $8 \times 16 \times 20 \mathrm{~mm}^{3}$ and filled with an aqueous solution of copper sulfate $\left(4 \times 10^{-3} \mathrm{M}\right)$ in order to accelerate the spin relaxation.

Volume imaging analysis of transparent foams: Starting point for the image analysis are the 3 - $\mathrm{d}$ images of the sponges, consisting of gray values for the $256^{3}$ voxels of the scan domain. Using this 3 -d volume data of transparent sponge, a model of the solid surface could be extracted with digital filter routines for noise reduction and an individual threshold value to separate solid from void. The selected threshold directly influences the surface area and, thus, simply selecting a value is somewhat vague as this threshold additionally depends on the operational parameters when acquiring the raw data. In the present work an automatic selection procedure was used that is described in detail in [20]. The whole procedure was programmed using the freely available Visualisation Toolkit (VTK) [21]. The results of the procedure are discretized surfaces comprising typically $0.5-1$ million connected tri- 
Citation: Jacobi A, Bucharsky EC, Schell KG, Habisreuther P, Oberacker R, Hoffmann MJ, et al. (2012) The Application of Transparent Glass Sponges for Improvement of Light Distribution in Photobioreactors. J Bioprocess Biotechniq 2:113 doi: 10.4172/2155-9821.1000113

angles with the exact number depending on the surface area and resolution. After discretization, calculating the surface areas can be done by a simple addition of these triangles areas.

In order to determine the porosity the void space on one side of the interface area can be filled with tetrahedral volume elements and again summed up to result in the volumes of the void phase. The tetrahedral meshes for this purpose were generated in this work using the commercially available meshing tool ANSYS ICEMCFD as this meshes can easily be imported by many simulation toolkits. In order to give an overview, in Figure 1 an image of the resulting geometry is shown.

Based on the detailed geometry of the samples an estimation of the extents of single pores has been derived. The proposed procedure starts with an estimation of points in the void phase that are assumed to be the pore centers. These points are determined by first calculating the Euclidean distance of every point in the void phase to the nearest surface point by application of the algorithm according to Saito and Toriwaki [22] and then searching local maxima in the 3-dimensional distance field. Twice of the local distance at these local maxima then gives an estimation of a minimal pore diameter. Using these values, a statistical analysis of the pore sizes was made. As a result, in Table 1 the average and the RMS values of the pore diameters as well as porosity and specific surface area for both 8 and 15 ppi glass sponges are given. Up to now, the influence of cell size of the foams on light distribution is not clear, but the structural parameters are intended to form the basic data for further analysis. These further studies are necessary, e.g. employing Monte-Carlo-Simulation of multiple light scattering, in order correlate the parameters to the light distribution.

\section{Cultivation in miniplate reactors}

Strains and medium: Chlamydomonas reinhardtii wildtype wt 13 [23] derived from the original Ebersold/Levine line 137c (CC-125 mt+) $[24,25]$ was used as a model organism. For maintenance and preculturing the algae was continuously grown mixotrophic in liquid cultures of TAP media (Tris Acetate Phosphate) [26] under low light intensities $\left(50 \mu \mathrm{E} \mathrm{m}^{-2} \cdot \mathrm{s}^{-1}\right)$.

Miniplate reactors: Special Miniplate reactors were developed for testing the transparent glass sponges (Figure 2). These consist of stainless steel frameworks (bottom and top plate, two side plates) with the internal measurements of $140 \times 100 \times 20 \mathrm{~mm}$ and two glass panes bonded between the side plates using neutral crosslinking silicon rubber (SCRINTEC 600, RALICKS Industrie- und Umwelttechnik, ReesHaldern, Germany). The bottom and top plate are sealed with a silicon gasket (1.5 mm VMQ-Elastomer, Angst + Pisterer, Zürich, Switzerland) and fixed with screws. For aeration and mixing a PTFE membrane ( $1 \mu \mathrm{m}$ THOMAPOR-Poröse Platte, RTC ReicheltChemietechnik, Heidelberg, Germany) is fixed in the bottom plate. The inlet and exhaust gas passes $0.2 \mu \mathrm{m}$ sterilefilters, the connection to the plate is done via LUER-LOCK. For sampling a syringe is used connected to a safe flow valve (B.Braun, Melsungen, Germany) and a needle. Light is supplied using 60 LEDs (MS6L083AT, Nichia, Japan) with $6^{\circ}$ collimating lenses (IMM Photonics GmbH, Unterschleißheim, Germany) in $100 \mathrm{~mm}$ distance from the illuminated side of the reactor.

Photoautotrophic cell growth was investigated in a working volume of $0.2 \mathrm{~L} 2.5 \mathrm{xTP}$ medium (TAP without Acetat), $\mathrm{pH} 8.8$ at varying light intensities. Inoculation was done after saturation of the medium with $\mathrm{CO}_{2}$ with a four days old preculture grown in TAP; the starting biomass was adjusted to approximately $0.05 \mathrm{~g} / \mathrm{L}$. The cell suspension was purged continuously with a sterile mixture of air enriched with $5 \% \mathrm{CO}_{2}$ at an aeration rate of $50 \mathrm{~mL} / \mathrm{min}$ controlled via two mass flow controllers (MassFlo, MKS Instruments, München, Germany). Temperature was kept constant at $25^{\circ} \mathrm{C}$ via an incubator (Flohr Instruments, The Netherlands). Light intensity was controlled using the control system BioProCon written as a routine in Lab View (National Instruments).

The installation of the glass sponges into the Miniplate reactor is shown schematically in Figure 6. The microalgae cells are grown in suspension within the pores of the sponges. The separation of algae after cultivation is easily done. The liquid suspension is just draining off from the solid sponges and thereby the algae.

\section{Analytics and calculations:}

- Biomass determination: Biomass concentrations were determined by offline optical density (OD) measurements at $750 \mathrm{~nm}$ using a spectrometer (T60 UV-Visible Spectrophotometer, PG instruments, Leicester, Great Britain).

A correlation between OD and biomass was established by dry weight measurements.

- Light measurements: Measurements of light intensity were done using a planar quantum sensor (MQS-C, Walz connected with a LI-250 Light Meter, Licor, Walz Mess- und Regelungstechnik, Effeltrich, Germany). Light intensities up to $1500 \mu \mathrm{E} \cdot \mathrm{m}^{-2} \cdot \mathrm{s}^{-1}$ were applied.

- Calculation of specific growth rate: The specific growth rate $\mu$ was determined in the exponential growth phase under nonlight limiting conditions (dry biomass $<0.2 \mathrm{~g} / \mathrm{L}$ ) according to the following formula.

$\frac{d x}{d t}=\mu_{\max } x$

with $\mathrm{x}$ biomass, $\mathrm{t}$ time and $\mu_{\max }$ maximum specific growth rate.

This can be converted to

$x=x_{0} \cdot e^{\mu_{\max } \cdot\left(t-t_{0}\right)}$
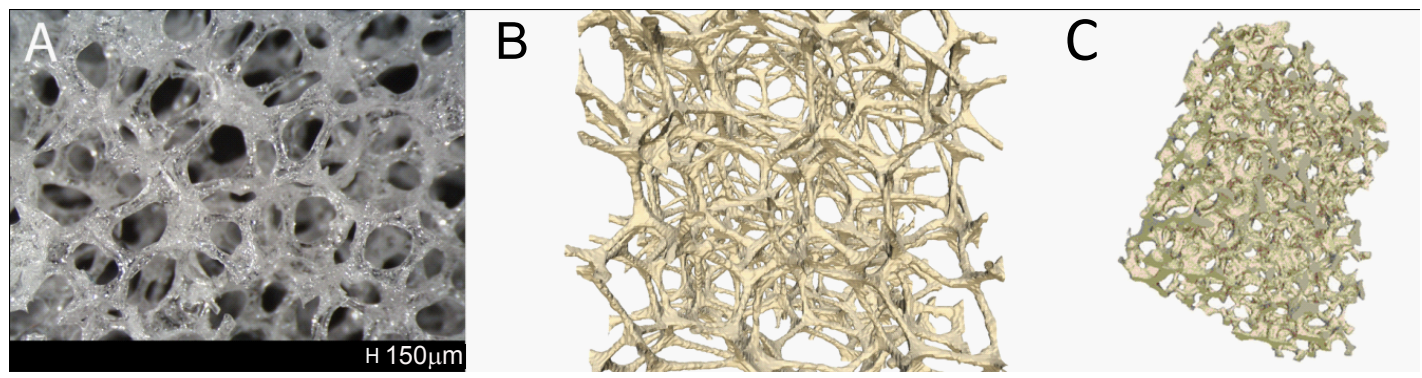

Figure 1: Real (A) and reconstructed (B, C) structure from 1HNMR image of the polymer template transparent sponge and sintered at $1330^{\circ} \mathrm{C}$. 
Citation: Jacobi A, Bucharsky EC, Schell KG, Habisreuther P, Oberacker R, Hoffmann MJ, et al. (2012) The Application of Transparent Glass Sponges for Improvement of Light Distribution in Photobioreactors. J Bioprocess Biotechniq 2:113 doi: 10.4172/2155-9821.1000113

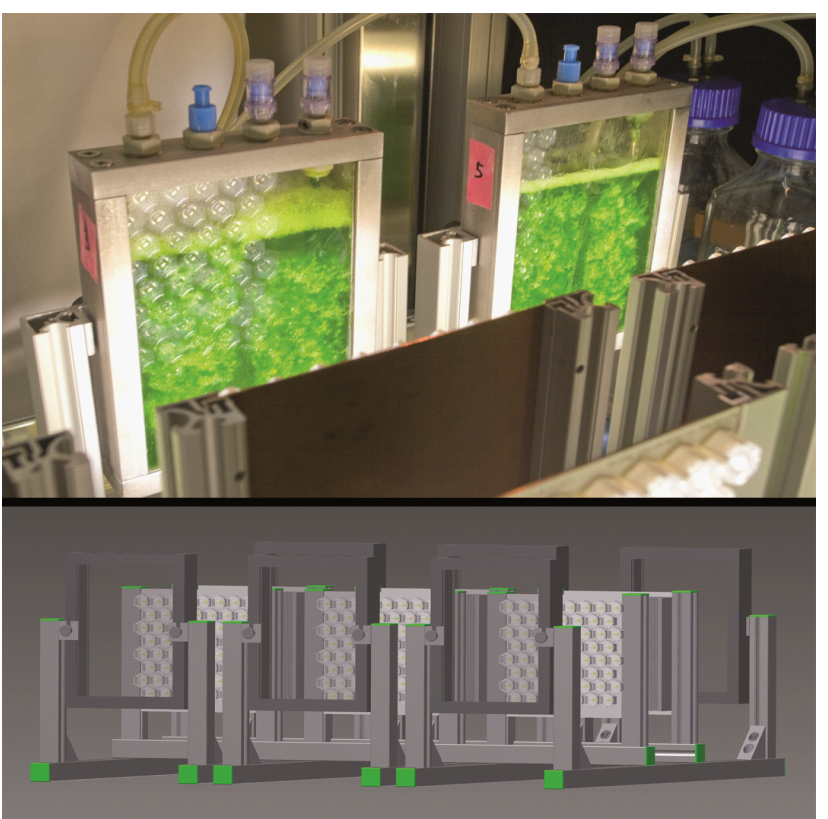

Figure 2: Mini-Plate Reactors as model system for testing transparent glass sponges.

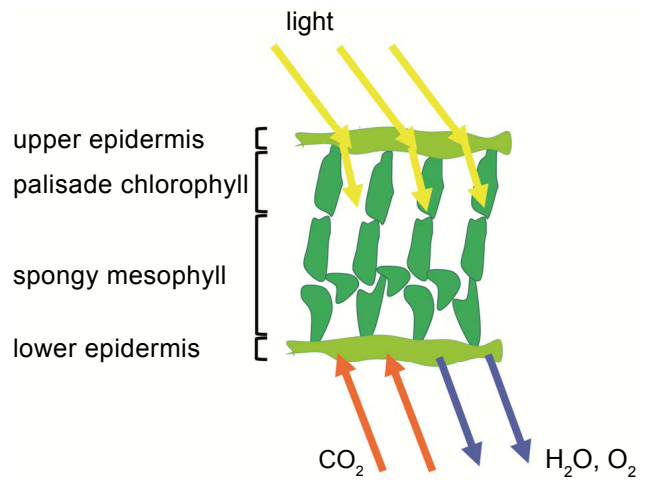

Figure 3: Schematic principle of leaf structure (leaf parenchyma) [10]

The values are averaged over at least three independent experiments.

- Calculation of photo conversion efficiency (PCE): The photo conversion efficiency (PCE) [J/J] describes the efficiency of biomass buildup form incident light energy. The following assumptions are used for calculation:

- biomass calorific value $20 \mathrm{MJ} / \mathrm{kg}[12,27]$

- light energy conversion factor $1 \mathrm{~W} / \mathrm{m}^{2}=4.75^{\star} \mu \mathrm{E} \cdot \mathrm{m}^{-2} \cdot \mathrm{s}^{-1}$ (calculated from spectral distribution of used LEDs, for PAR photosynthetic active radiation $400-700 \mathrm{~nm}$ )

Finally a PCE based on PAR was calculated during the linear, light limited growth phase at higher cell densities (dry biomass $<1 \mathrm{~g} / \mathrm{L}$ ) for defined light intensities. The values are also averaged over at least three independent experiments.

\section{Results and Discussion}

\section{Anticipated effects of sponges for light dilution}

The enlargement of the illuminated surface of photobioreactors is the main intention today to improve the efficiency of phototrophic cultivation systems. Data for SVR and SFR ratios of photobioreactors and open ponds are given in Table 2. "Natural photosynthetic systems" like trees could reach ten times higher values (Table 2). This can be explained by the "lime tree model" communicated by Pulz: the photosynthetic active leaf surface area accounts for $2500 \mathrm{~m}^{2}$ and the footprint area for only $100 \mathrm{~m}^{2}$. But comparing the illuminated surface area of photobioreactors with the leaf area of trees is only the first step for improvements.

The second step is based on an idea of nature inspired design published by Posten [10]. An additional effect on efficiency could be reached by enlargement of the inner surface of the reactor for light dilution and supply. Similar inner structures can be found in leafs of trees called the parenchyma (Figure 3). For this purpose transparent, open pored glass sponges are used as installations in photobioreactors. By inserting transparent $8 \mathrm{ppi}$ sponges as light diluting materials into the reactor the SVR can be increased to a value of $568 \mathrm{~m}^{-1}$ (see Table 1). An additional effect of the sponges would be the reduction of light gradients inside the whole reactor volume. Based on this concept photobioreactors still have quite promising potential for further developments.

The necessity of light dilution and the reduction of light gradients in photobioreactors can be explained with the growth kinetics of microalgae which show the growth rate depending on light intensity. Ex-

\begin{tabular}{|l|c|c|c|}
\hline & & 8 ppi(glass) & $15 p p i(g l a s s)$ \\
\hline Porosity & - & 0.90 & 0.86 \\
\hline Specific surface area & $\mathrm{m}^{-1}$ & 568 & 883 \\
\hline RMS pore diameter & $\mathrm{mm}$ & 0.24 & 0.2073 \\
\hline average pore diameter & $\mathrm{mm}$ & 3.56 & 2.79 \\
\hline
\end{tabular}

Table 1: Foam parameters, obtained from volume analysis.

\begin{tabular}{|l|l|l|l|}
\hline & Lime tree & PBR & Open Pond \\
\hline SVR $/ \mathrm{m}^{2} / \mathrm{m}^{3}$ & $<1000$ & $<100$ & $<4$ \\
\hline $\mathrm{SFR} / \mathrm{m}^{2} / \mathrm{m}^{2}$ & $<25$ & $<10$ & 1 \\
\hline
\end{tabular}

Table 2: Values for surface to volume ratios (SVR) and surface to footprint ratios (SFR) for the lime tree model, photobioreactors and open ponds.

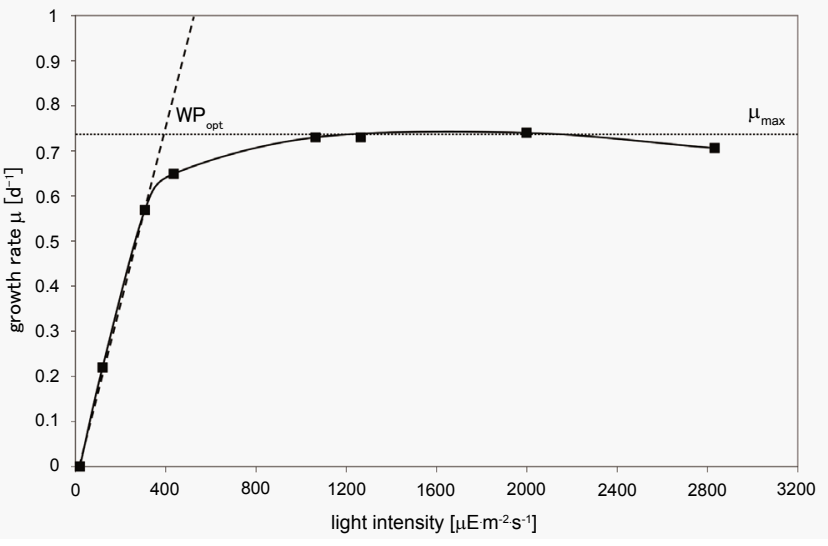

Figure 4: Growth kinetic of Porphyridium purpureum (modified based on [10]), with $\mu_{\max }$ maximum growth rate and $W P_{\text {opt }}$ optimal and most efficient working point of biomass production with highest increase of growth with rising light intensity. 
Citation: Jacobi A, Bucharsky EC, Schell KG, Habisreuther P, Oberacker R, Hoffmann MJ, et al. (2012) The Application of Transparent Glass Sponges for Improvement of Light Distribution in Photobioreactors. J Bioprocess Biotechniq 2:113 doi: 10.4172/2155-9821.1000113

Page 5 of 8

emplarily the growth kinetic of the red algae Porphyridium purpureum is displayed in Figure 4. The different effects of light intensity on microalgal growth can be observed from this data. Growth is light limited at low light intensities up to about $400 \mu \mathrm{E} \cdot \mathrm{m}^{-2} \cdot \mathrm{s}^{-1}$. Then the optimal and most efficient working point of biomass production is reached with highest increase of growth with rising light intensity. At light intensities $>400 \mu \mathrm{E} \cdot \mathrm{m}^{-2} \cdot \mathrm{s}^{-1}$ photosynthesis becomes light saturated and later even inhibited. That means the more light energy supplied to the cultures cannot be stored in biomass or products.

The low efficiencies of production systems today is the combined effect of different light conditions outside and inside the reactors on growth increased even due to the change of optical densities of the algae suspension during the cultivation process. Figure 5 depicts schematically conditions in plate reactors illuminated from one side at changing cell densities. Only at low cell concentrations when all algae obtain sufficient light for growth the maximum growth rate $\mu_{\text {mx }}$ will be reached. With increasing optical density of the suspension volume elements with light limited conditions $\left(\mu_{\lim }\right)$ or even no cell growth $(\mu=0)$ will exist. The overall growth rate measured under this condition will be a combination of all these effects.

For optimization of light dilution in photobioreactors transparent open pored sponges could be used as installations (for a schema see Figure 6). The algae grow in suspension within the pores of the sponge. The sponge structure dilutes light into the deeper, dark parts of the reactor by light conduction in the struts, as well as reflection and refraction at the surfaces due to the refractive index of glass which is higher than those of water. Therefore an amorphous $\mathrm{SiO}_{2}$ glass structure has to be produced. Ideally sun light should be diluted to the optimal light intensity in the whole cultivation volume. Thereby the efficiency of production scale rectors could be improved by maximizing overall growth rates.
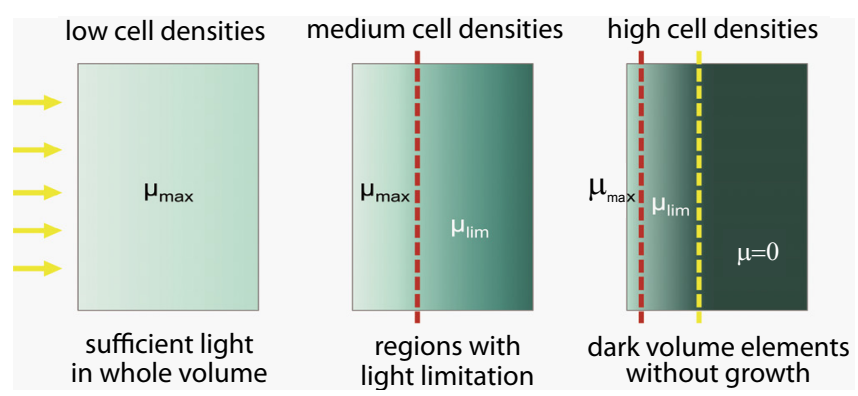

Figure 5: Schema of light conditions in a plate photobioreactor illuminated from one side at different cell densities, with $\mu_{\max }$ maximum growth rate, $\mu_{\text {lim }}$ light limited growth rate, $\mu=0$ no cell growth.

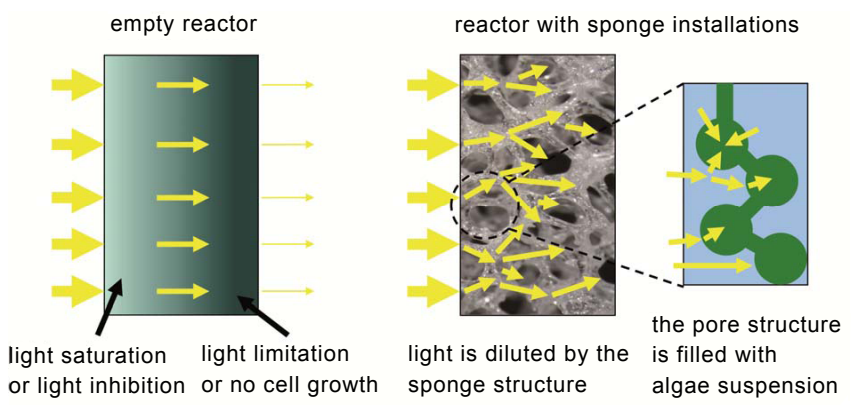

Figure 6: Installation of glass sponges into Mini-Plate Reactors, Schema of light dilution.

\section{Preparation and characterization of transparent open-pored structures}

Suspension: stability and viscosity: In order to identify a suitable $\mathrm{pH}$-range for electrostatic stabilization of the silica particles $\zeta$-potential measurements were performed [13]. Low-viscosity slurries are necessary for coating the polymer. In the system $\mathrm{SiO}_{2}$ - water a compromise between a maximum of $\zeta$ - potential and suitable viscosity was obtained at a $\mathrm{pH}$ level higher than $\mathrm{pH} 10$.

Using well stabilized slurries, an increase of $\mathrm{SiO}_{2}$ leads to a moderate increase of viscosity and therefore a maximal concentration with acceptable viscosity values was obtained for slurries with solids loadings up to $\Phi<60 \mathrm{wt} \%$.

Coated, dryer and burning out the polymer template: The aim of the impregnation process is to achieve a coating layer of defined thickness on the polymer template, consisting of dense and homogenously packed $\mathrm{SiO}_{2}$ nanoparticles. Both, structure and thickness of the layer depend strongly on the suspension used. The dominating parameters which control these factors are the solids loading, the interparticle forces and as consequence the viscosity. With respect to a sufficient coating layer thickness, a high solids loading is desirable. A dense and homogeneous particle packing requires repulsive interparticle forces. Both can be achieved by adjusting the $\mathrm{pH}$-value as discussed previously.

Burning out the polymer is a very important step for obtaining manageable green bodies. A special temperature regimen has to be applied and a sufficient concentration of $\mathrm{SiO}_{2}$ on the struts is necessary to prevent the framework from collapsing. Therefore, the solids loading of the slurry should be high.

Regardless the used slurry, the recommended amount of $\mathrm{SiO}_{2}$ should be higher than approximately 0.1 grams per cubic centimeter of template. Below this value for the polymer employed in this study [ 8 and $15 \mathrm{ppi}$ ] it was not possible to get the desired structure through the burning-out process [28]. But if the thermal treatment is successful, there is no significant change in structure visible to the naked eye and the color of the samples remain white.

Figure 7 depicts a coated sample before (A) and after burning out the polymeric framework (B). It is worth to note, that the geometrical density of the shown greenbody was $>0.1 \mathrm{~g} / \mathrm{cm}^{3}$. In the case of a density of $0.08 \mathrm{~g} / \mathrm{cm}^{3}$, see Figure 8 , the framework was pulverized during the burning-out step.

Sintering and characterization: After the burning-out step, the obtained green bodies can be densified by viscous-flow sintering, leading to shrinkage of about $20 \%$ in all directions. By carefully selecting the sintering regime, i.e. an appropriate heating rate, temperature and dwell time, the initial white green bodies can be transferred into transparent open-celled foams. Transparency indicates that the amorphous character of the initial powder is still present. To monitor the phase composition XRD spectra were performed after sintering at temperatures varying from 1150 to $1400^{\circ} \mathrm{C}$. In Figure 9, typical XRD-diffraction patterns of amorphous $\mathrm{SiO}_{2}$ after sintering at $1350^{\circ} \mathrm{C}$ are shown. Above this temperature, $\mathrm{SiO}_{2}$ foams commence to crystallize, as indicated in the XRD- spectra (Figure 9). Hence, after sintering at $1400^{\circ} \mathrm{C}$ the XRD pattern corresponds to crystalline cristobalite as the only detectable phase.

The broad peak near $22^{\circ}$ is attributed to the short-range order in amorphous silica, whereas the sharp peaks in Figure 9 (right side) show the formed crystalline structure of cristobalite. 
Citation: Jacobi A, Bucharsky EC, Schell KG, Habisreuther P, Oberacker R, Hoffmann MJ, et al. (2012) The Application of Transparent Glass Sponges for Improvement of Light Distribution in Photobioreactors. J Bioprocess Biotechniq 2:113 doi: 10.4172/2155-9821.1000113

Page 6 of 8

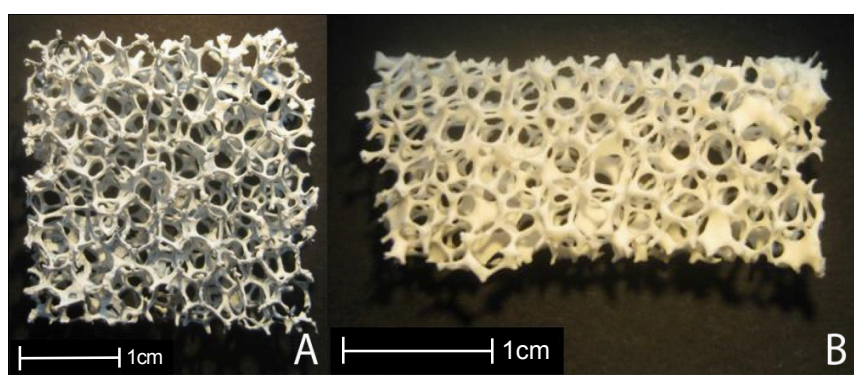

Figure 7: Coated sample before $(A)$ and after burning out the polymeric framework $(B)$, geometrical density of the greenbody $>0.1 \mathrm{~g} / \mathrm{cm}^{3}$.

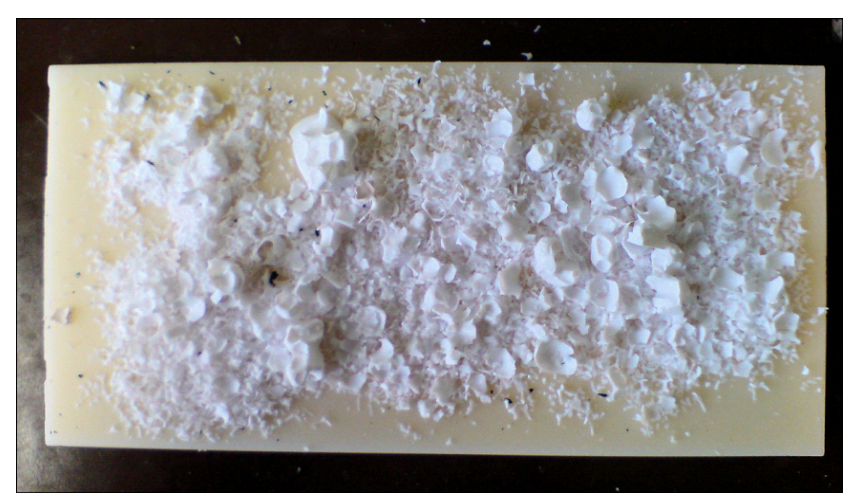

Figure 8: Pulverization of the framework during the burning-out step, geometrical density of the greenbody $0.08 \mathrm{~g} / \mathrm{cm}^{3}$.

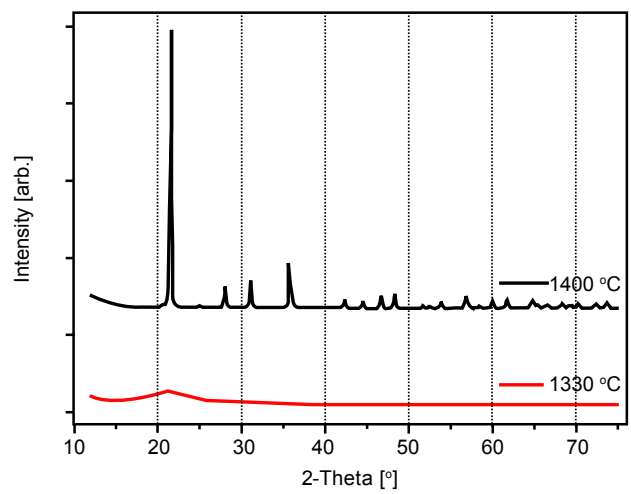

Figure 9: Typical XRD-diffraction patterns of amorphous $\mathrm{SiO}_{2}$ after sintering at $1350^{\circ} \mathrm{C}$

Additionally to the XRD spectra, changes in structure and consequently on the transparency can be explained considering the apparent density and SEM analysis. These topics were extensively discussed in our previous work [28].

\section{Cultivation experiments/ Determination of growth rates and photo conversion efficiencies (PCE)}

The aim of experiments performed in Miniplate reactors is to test the transparent glass sponges as light diluting and light distributing installations in photobioreactors. Relevant parameters to estimate in this context are the growth rate $\mu$ in the exponential growth phase at low cell densities and non-limiting conditions and more important the photo conversion efficiency (PCE) at high cell densities. The latter is a measure for the efficiency of biomass buildup from the captured light energy.

The reference system is the "empty" reactor applied for growth experiments without any light distributing installations inside. To increase the final cell densities reached in the reactor already concentrated medium (2.5xTP) is used at the beginning of the cultivation with $\mathrm{CO}_{2}$ supply by aeration (5\%). Typical growth curves of batch experiments at a selected light intensity $\left(500 \mu \mathrm{E} \cdot \mathrm{m}^{-2} \cdot \mathrm{s}^{-1}\right)$ and the corresponding light transmission behind the reactor for 8 and 15 ppi glass sponges and the empty reactor are shown in Figure 10.

A characteristic growth curve of micro algal batch cultures is observed with a very short exponential phase until day one at low cell densities $<1 \mathrm{~g} / \mathrm{L}$. Already at day one light becomes limiting but in contrast to other substrates it constantly further supplied which results in a transition into a linear growth phase. When Ammonium is limiting in the medium (ionchromatography measurements not shown) the maximum biomass concentration of about 3-3.5 g/L is reached during the third day.

The light transmission on the non-illuminated side of the reactor decreases in correlation with the increasing biomass concentrations resulting in light limiting conditions with transmission equals zero at day one. The light transmission at the beginning of the cultivation is lower with sponge filled reactors compared to the empty one. This is possibly due to a better light distribution inside the reactor caused by refraction and scattering. It could be a first indication for a better light dilution in the reactor by the sponge structure. It is also possible that by scattering at the sponge surface some amount of light is lost at the top, bottom and the small dark sides of the reactor. This amount is potentially bigger than in the empty reactor but could not be measured. The portion of scattering due to cells would be equal in all three systems. The measured reflection at the illuminated surface is less than $<10 \%$ of the incident light intensity and no difference between the different configurations (empty or sponge filled reactor) can be observed. The influence of reflection at the reactor surface (typically $4 \%$ for air-glass) on incident light energy is eliminated by calibrating inside the reactor

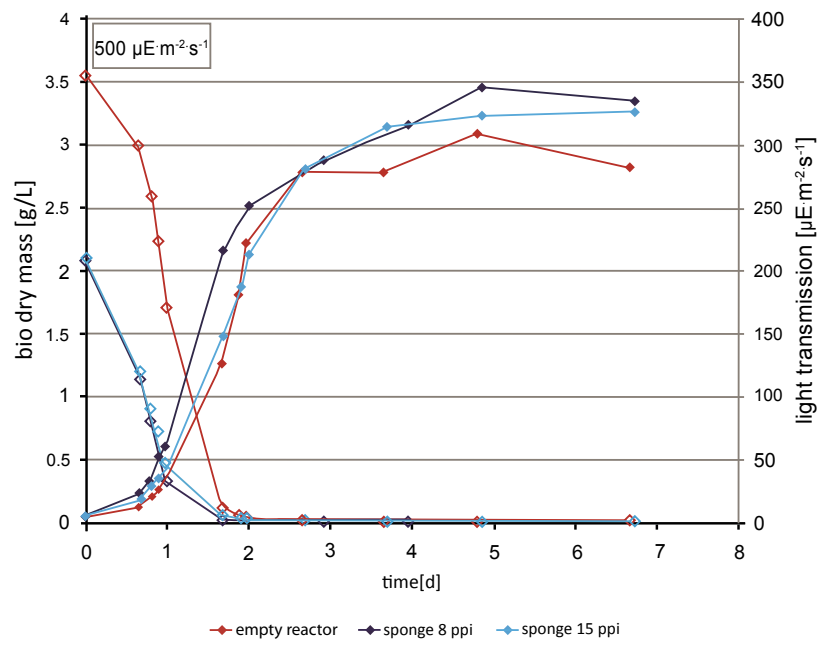

Figure 10: Bio dry mass concentration of wt13 and light transmission ove cultivation time for 8 and 15 ppi glass sponges and the empty reactor 500 $\mu \mathrm{E} \cdot \mathrm{m}^{-2} \cdot \mathrm{s}^{-1}, 2.5 \mathrm{xTP}, 5 \% \mathrm{CO}_{2}$ supply by aeration. 
Citation: Jacobi A, Bucharsky EC, Schell KG, Habisreuther P, Oberacker R, Hoffmann MJ, et al. (2012) The Application of Transparent Glass Sponges for Improvement of Light Distribution in Photobioreactors. J Bioprocess Biotechniq 2:113 doi: 10.4172/2155-9821.1000113

behind the first glass plate. The absorption of light by glass is only in the rage of $<0.1 \%$ per $m$ light path.

Growth rates $\mu$ are determined in the exponential growth phase (until day one) and the resulting growth kinetic of Chlamydomonas wildtype depending on the applied light intensity in reactors with sponges as light diluting structures (pore size 8 and 15 ppi) compared to the empty reactor is depicted in Figure 11.

The growth kinetic of Chlamydomonas wt13 (empty reactor) illustrates the three possible light regimes in an algal culture. Light intensity is limiting until intensities up to $200-250 \mu \mathrm{E} \cdot \mathrm{m}^{-2} \cdot \mathrm{s}^{-1}$ and the growth rate $\mu$ increases straight linear with rising light energy. At light intensities $>400 \mu \mathrm{E} \cdot \mathrm{m}^{-2} \cdot \mathrm{s}^{-1}$ light becomes saturating and the additional energy cannot be used for photosynthesis. At even higher intensities $\geq 1000$ $\mu \mathrm{E} \cdot \mathrm{m}^{-2} \cdot \mathrm{s}^{-1}$ the inhibiting effect of too much light can already be observed resulting in a decrease of growth rate $\mu$.

The effect of the glass sponges was tested at 200, 500, 800 and 1000 $\mu \mathrm{E} \cdot \mathrm{m}^{-2} \cdot \mathrm{s}^{-1}$ and a positive influence on the growth rates $\mu$ (about $+25 \%$ ) at low cell densities can be seen.

But more interesting would be the effect at higher cell concentrations. The photo conversion efficiency (PCE) can be used as a measure to assess the use of transparent glass sponges as installations in photobioreactors. The calculation can be done during the linear growth phase under light limiting conditions from the increase of biomass and the applied light intensity. However one major limiting factor is the medium and the short linear growth phase because of ammonium limitation at day two. Therefore optimization of medium composition and testing of different feeding strategies are in progress to increase final biomass concentrations and prolong the linear growth phase.

First calculations of PCEs at $500 \mu \mathrm{E} \cdot \mathrm{m}^{-2} \cdot \mathrm{s}^{-1}$ were done using a biomass calorific value of $20 \mathrm{MJ} / \mathrm{kg}$ [12,27] and a light energy conversion factor of $1 \mathrm{~W} / \mathrm{m}^{2}=4.75^{\star} \mu \mathrm{E} \cdot \mathrm{m}^{-2} \cdot \mathrm{s}^{-1}$ (calculated from spectral distribution of used LEDs, for PAR photosynthetic active radiation 400-700 $\mathrm{nm})$. The results indicate an increase from $4.9 \%$ for the empty reactor to $5.6 \%$ and $5.9 \%$ for the 8 and 15 ppi sponge filled reactor. These are promising results with potential for further improvement.

\section{Concluding remarks}

It has been shown that the new concept for improving light dilu-

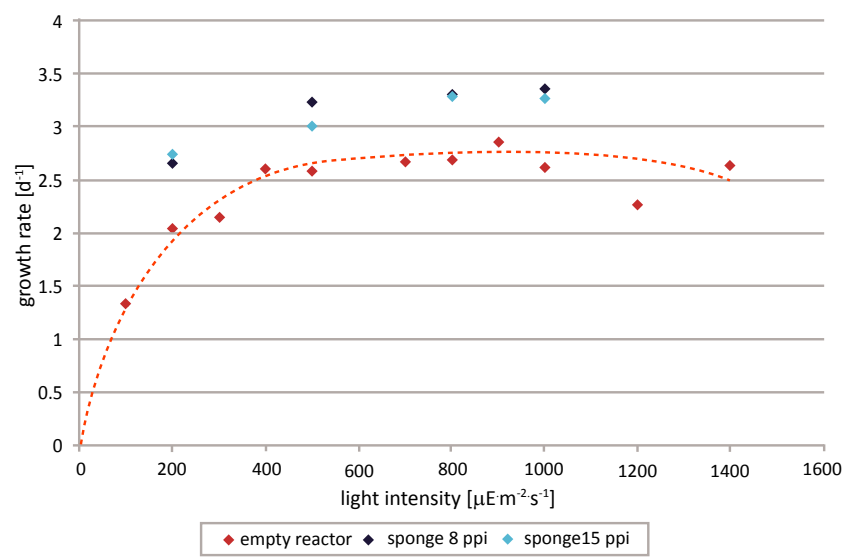

Figure 11: Growth kinetics of Chlamydomonas reinhardtii wt13 depending on light intensity determined in the Miniplate reactor with 8 and 15 ppi glass sponges as light conducting installations and the empty reactor for comparison in the exponential growth phase at low cell densities $<1 \mathrm{~g} / \mathrm{L}$. tion and light distribution in photobioreactors by applying transparent, open-pored sponges was realized and tested during cultivation experiments.

A manufacturing process based on the polymer replica technique was established and important factors influencing the process to get stable and transparent probes like slurry stabilization (viscosity, zetapotential, $\mathrm{pH}$ ), sintering temperature and shrinkage were investigated. The 8 ppi sponge shows the following characteristics: porosity 0.9 , specific surface area $568 \mathrm{~m}^{-1}$ which means an increase of the (inner) surface to volume ratio of the reactor.

Cultivation experiments showed an increase in growth rate (about $+25 \%)$ at low cell densities and the PCE was increased from $4.9 \%$ for the empty reactor to $5.6 \%$ and $5.9 \%$ for the 8 and 15 ppi sponge filled reactor. The results show a first positive effect, but further progress is necessary. The effect will be more apparent at higher light intensities and higher cell densities. Therefore experiments to increase biomass concentrations by concentrating the TP medium ( 2.5 fold) and appropriate feeding are in progress. Furthermore a scale-up to larger volumes would be necessary to mimic conditions in production reactors which is limited to the producible sponge size at the moment.

The final target would be thin reactor systems with internal light diluting structures lying horizontally on the ground and supplying $\mathrm{CO}_{2}$ by surface or membrane aeration. This would diminish cost for materials and supporting installations due to less water demand and decreased resulting hydrostatic pressure. Furthermore the need of auxiliary energy can be reduced when aeration and gas hold up are minimized.

\section{Acknowledgements}

The authors would like to thank the German Research Foundation (DFG) for funding the Research Group FOR 583 "Solid Sponges-Application of monolithic network structures in process engineering."

We acknowledge support by Deutsche Forschungsgemeinschaft and Open Access Publishing Fund of Karlsruhe Institute of Technology.

\section{References}

1. Pulz O, Gross W (2004) Valuable products from biotechnology of microalgae Appl Microbiol Biotechnol 65: 635-648.

2. Rosello Sastre R, Posten C (2010) Die vielfältige Anwendung von Mikroalgen als nachwachsende Rohstoffe. The Variety of Microalgae Applications as a Renewable Resource. Chem Ing Tech 82: 1925-1939.

3. Wijffels RH, Barbosa MJ (2010) An Outlook on Microalgal Biofuels. Science 329: 796-799

4. Rosenberg JN, Oyler GA, Wilkinson L, Betenbaugh MJ (2008) A green light for engineered algae: redirecting metabolism to fuel a biotechnology revolution. Curr Opin Biotechnol 19: 430-436.

5. Dismukes GC, Carrieri D, Bennette N, Ananyev GM, Posewitz MC (2008) Aquatic phototrophs: efficient alternatives to land-based crops for biofuels. Curr Opin Biotechnol 19: 235-240.

6. Blankenship RE, Tiede DM, Barber J, Brudvig GW, Fleming G, et al. (2011) Comparing Photosynthetic and Photovoltaic Efficiencies and Recognizing the Potential for Improvement. Science 332: 805-809.

7. Carvalho AP, Silva SO, Baptista JM, Malcata FX (2011) Light requirements in microalgal photobioreactors: an overview of biophotonic aspects. Appl Microbiol Biotechnol 89: 1275-1288.

8. Tredici MR (2010) Photobiology of microalgae mass cultures: understanding the tools for the next green revolution. Biofuels 1: 143-162.

9. Xu L, Weathers PJ, Xiong XR, Liu CZ (2009) Microalgal bioreactors: Challenges and opportunities. Eng Life Sci 9: 178-189. 
Citation: Jacobi A, Bucharsky EC, Schell KG, Habisreuther P, Oberacker R, Hoffmann MJ, et al. (2012) The Application of Transparent Glass Sponges for Improvement of Light Distribution in Photobioreactors. J Bioprocess Biotechniq 2:113 doi: 10.4172/2155-9821.1000113

Page 8 of 8

10. Posten C (2009) Design principles of photo-bioreactors for cultivation of microalgae. Eng Life Sci 9: 165-177.

11. Morweiser M, Kruse O, Hankamer B, Posten C (2010) Developments and perspectives of photobioreactors for biofuel production. Appl Microbiol Biotechnol 87: 1291-1301.

12. Lehr F, Posten C (2009) Closed photo-bioreactors as tools for biofuel production. Curr Opin Biotechnol 20: 280-285.

13. Bucharsky EC, Schell KG, Oberacker R, Hoffmann MJ (2010) Preparation of transparent glass sponges via replica method using high-purity silica. J Am Ceram Soc 93: 111-114.

14. Schwartzwaelder K, Sommer AV, Mich F (1963) Method of making porous ceramic particles. United States, Patent No. 3090094

15. Timur A (1969) Pulsed nuclear magnetic resonance studies of porosity, movable fluid, and permeability of sandstones. J Petrol Technol 21: 775-786.

16. Fredrich JT, Menendez B, Wong TF (1995) Imaging the pore structure of geomaterials. Science 268: 276-279.

17. Petrasch J, Wyss P, Steinfeld A (2007) Tomography-based Monte Carlo determination of radiative properties of reticulate porous ceramics. J Quant Spectrosc Radiat Transf 105: 180-197.

18. Coles ME, Hazlett RD, Spanne P, Soll WE, Muegge EL, et al. (1998) Pore level imaging of fluid transport using synchroton X-ray microtomography. J Petrol Sci Eng 19: 55-63.

19. Habisreuther P, Djordjevic N, Zarzalis N (2009) Statistical distribution of resi- dence time and tortuosity of flow through open-cell foams. Chem Eng Sci 64 4943-4954.

20. Grosse J, Dietrich B, Garrido GI, Habisreuther P, Zarzalis N, et al. (2009) Morphological Characterization of Ceramic Sponges for Applications in Chemical Engineering. Ind Eng Chem Res 48: 10395-10401.

21. Kitware I (2006) The VTK users guide. Kitware Inc., Colombia, USA.

22. Saito T, Toriwaki JI (1994) New algorithms for euclidean distance transformation of an n-dimensional digitized picture with applications. Pattern Recogn 27 1551-1565.

23. Schönfeld C, Wobbe L, Borgstädt R, Kienast A, Nixon PJ, et al. (2004) The Nucleus-encoded Protein MOC1 Is Essential for Mitochondrial Light Acclimation in Chlamydomonas reinhardtii. J Biol Chem 279: 50366-50374.

24. Harris EH (2001) Chlamydomonas as a model organism. Annu Review Plant Physiol Plant Mol Biol 52: 363-406.

25. Pröschold T, Harris EH, Coleman AW (2005) Portrait of a Species: Chlamydomonas reinhardtii. Genetics 170: 1601-1610.

26. Harris EH (2009) The Chlamydomonas sourcebook: Introduction to chlamydomonas and its laboratory use. ( ${ }^{\text {nd }}$ edn.), Academic Press, Amsterdam.

27. Schaub G, Vetter A (2007) Biofuels - a review. Chem Ing Tech 79: 569-578.

28. Bucharsky EC, Schell KG, Habisreuther P, Oberacker R, Zarzalis N, et al (2011) Preparation of Optically Transparent Open-Celled Foams and its Morphological Characterization Employing Volume Image Analysis. Adv Eng Mater 13: $1060-1065$
Submit your next manuscript and get advantages of OMICS Group submissions

Unique features:

User friendly/feasible website-translation of your paper to 50 world's leading languages

Audio Version of published paper

Digital articles to share and explore

Special features:

200 Open Access Journals

15,000 editorial tean

21 days rapid review process

Quality and quick editorial, review and publication processing

Indexing at PubMed (partial), Scopus, DOAJ, EBSCO, Index Copernicus and Google Scholar etc

Sharing Option: Social Networking Enabled

- Authors, Reviewers and Editors rewarded with online Scientific Credits

Better discount for your subsequent articles

Submit your manuscript at: www.omicsonline.org/submission 